\title{
Mono-sized sphere packing algorithm development using optimized Monte Carlo technique
}

Karn Soontrapa and Yitung Chen

Department of Mechanical Engineering, University of Nevada Las Vegas, NV 89154-4054

\begin{abstract}
In this research, fuel cell catalyst layer was developed using the optimized sphere packing algorithm. An optimization technique named adaptive random search technique (ARSET) was employed in this packing algorithm. The ARSET algorithm will generate the initial location of spheres and allow them to move in the random direction with the variable moving distance, randomly selected from the sampling range (a), based on the Lennard-Jones potential and Morse potential of the current and new configuration. The solid fraction values obtained from this developed algorithm are in the range of $0.610-0.624$ while the actual processing time can significantly be reduced by $5.58-34 \%$ based on the number of spheres. The initial random number sampling range (a) was investigated and the appropriate a value is equal to 0.5 .
\end{abstract}

\section{Background}

To understand the chemical reaction that happened in PEMFC, catalyst layer's structure was developed using the sphere packing process. Spheres in this packing process will represent carbon atoms because carbon is the common material for the catalyst layer.

Minimization of the total potential energy is the key for this packing process and the total potential energy is the combination between

the Lennard-Jones potential and the Morse potential

$L\left(r_{i j}\right)=4 \varepsilon\left[\left(\sigma / r_{i j}\right)^{12}-\left(\sigma / r_{i j}\right)^{6}\right]$

$M\left(r_{i j}\right)=\varepsilon\left[e^{-2 \alpha\left(r_{i j}-r_{0}\right)}-2 e^{-\alpha\left(r_{i j}-r_{0}\right)}\right]$

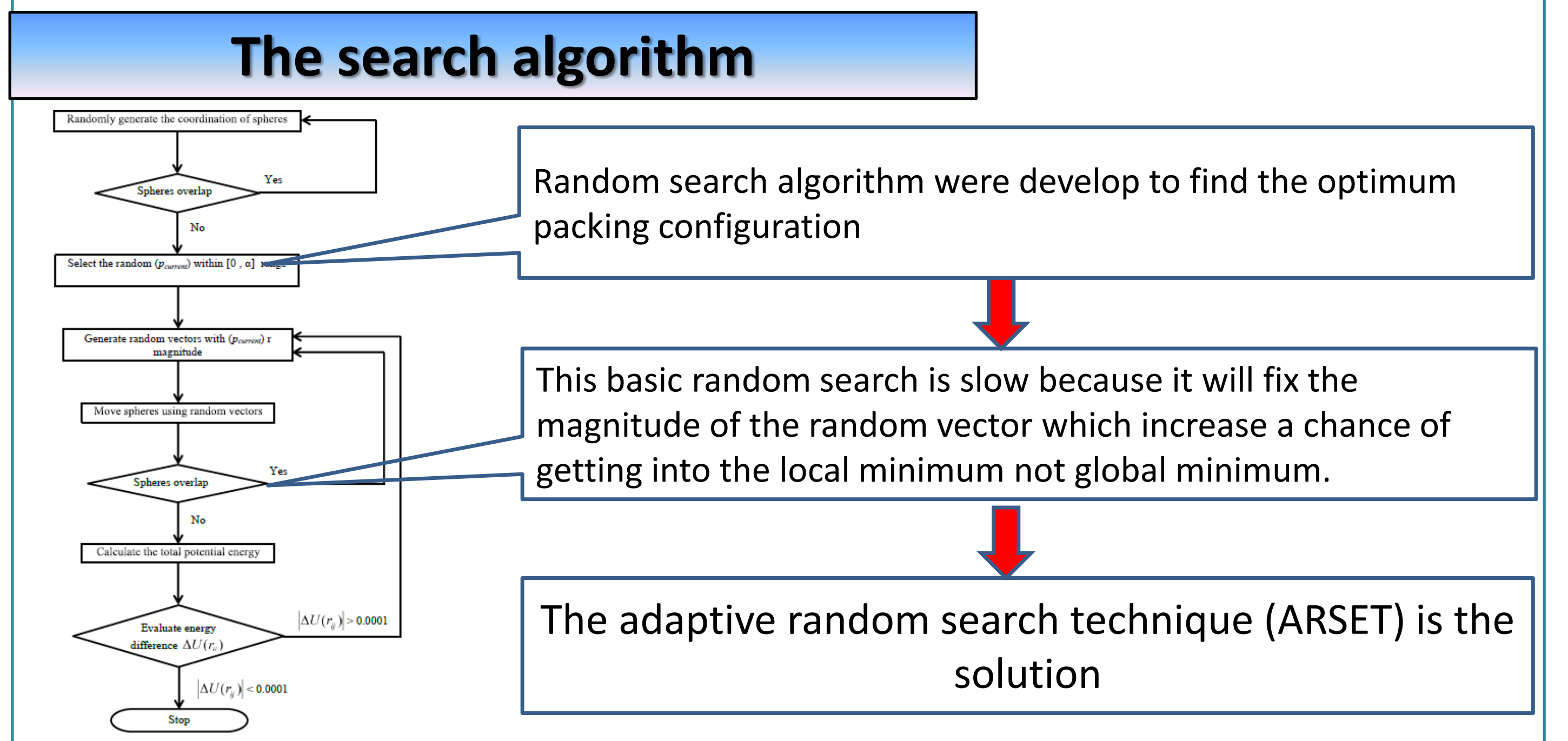

\section{Flow chart of the ARSET algorithm}

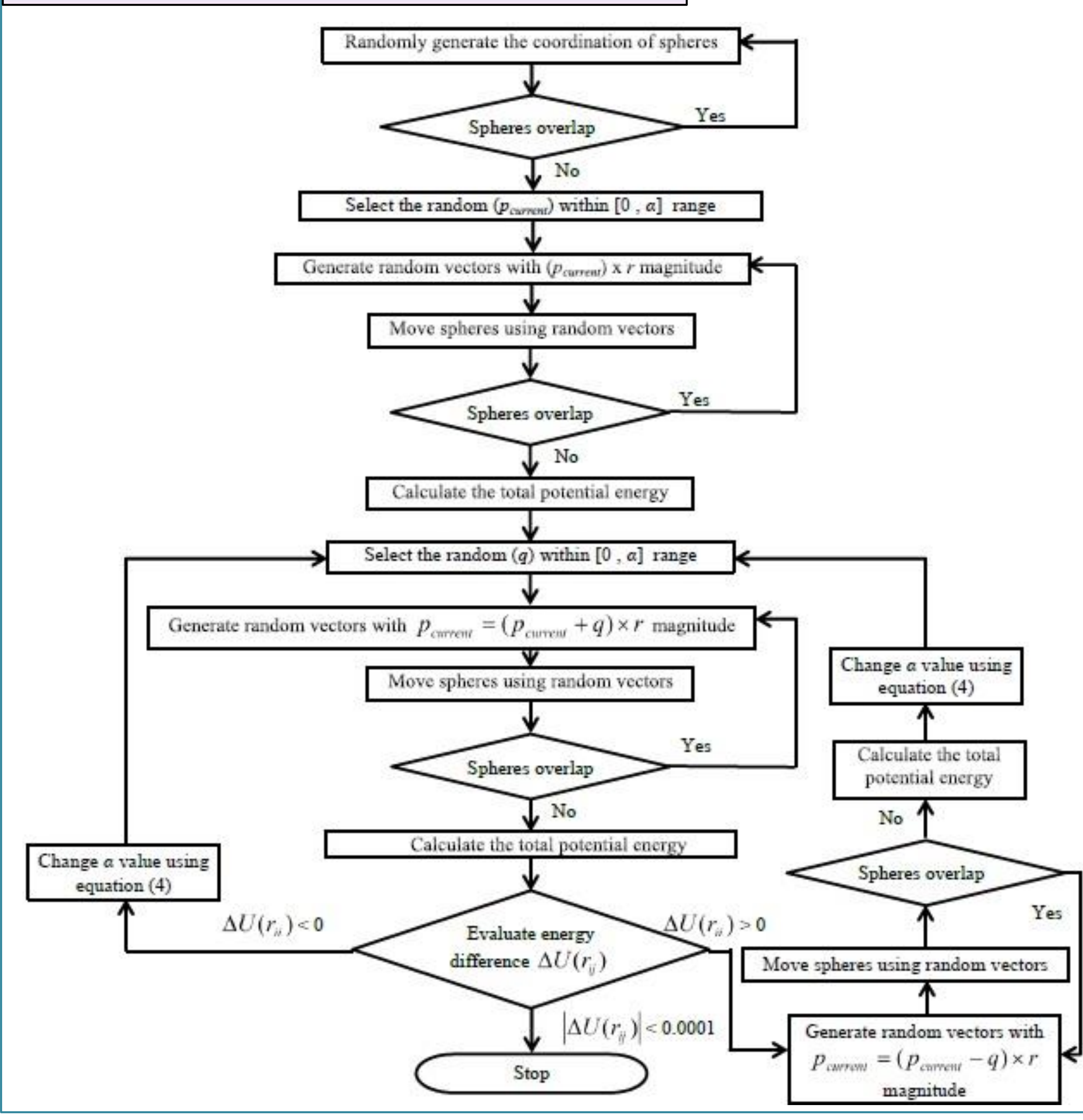

\section{Result and Discussion}

Magnitude of the random number and its effect

In the ARSET algorithm, spheres will allow to move along the random vector while the magnitudes of these random numbers are limited by the value of $\alpha$.
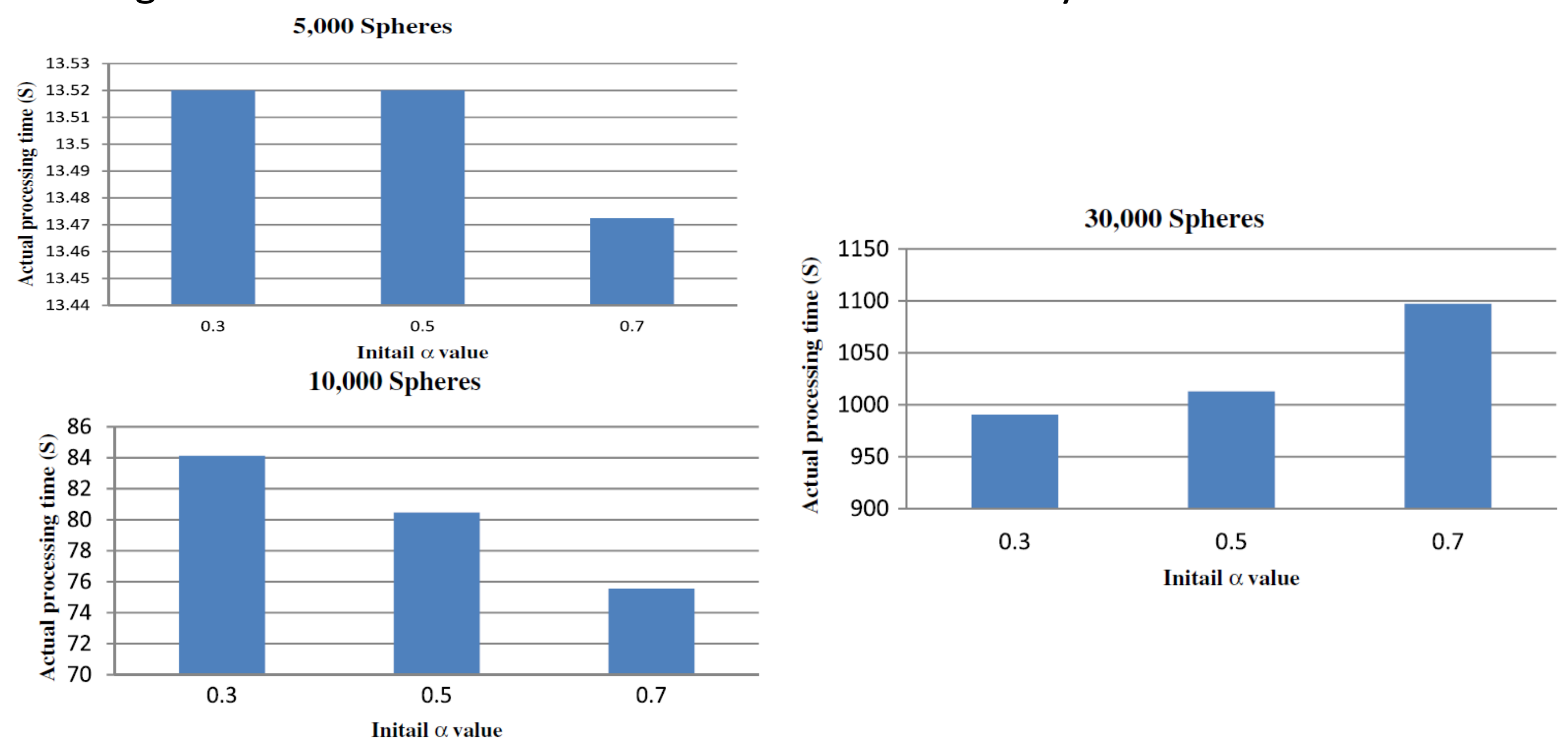

We suggest using the moderate initial a value like 0.5 to be the universal initial a value because it gives moderate processing time for 5000 , and 30,000 spheres cases and the shortest processing time for 10,000 spheres.

\begin{tabular}{|lll}
\hline \multicolumn{2}{|c|}{ Simulation Result } \\
\cline { 1 - 2 } Actual processing time comparison between BRST and ARSET algorithm. \\
\hline Number of spheres & \multicolumn{1}{c}{ Actual processing time $(\mathrm{s})$} \\
\cline { 2 - 3 } & BRST & ARSET (\% difference) \\
\hline 1000 & 0.39 & $0.37(-5.58 \%)$ \\
1800 & 0.58 & $0.50(-15 \%)$ \\
5000 & 16.81 & $13.05(-22 \%)$ \\
10,000 & 98.34 & $64.32(-34 \%)$ \\
30,000 & 1308.73 & $936.81(-28.42 \%)$ \\
\hline
\end{tabular}

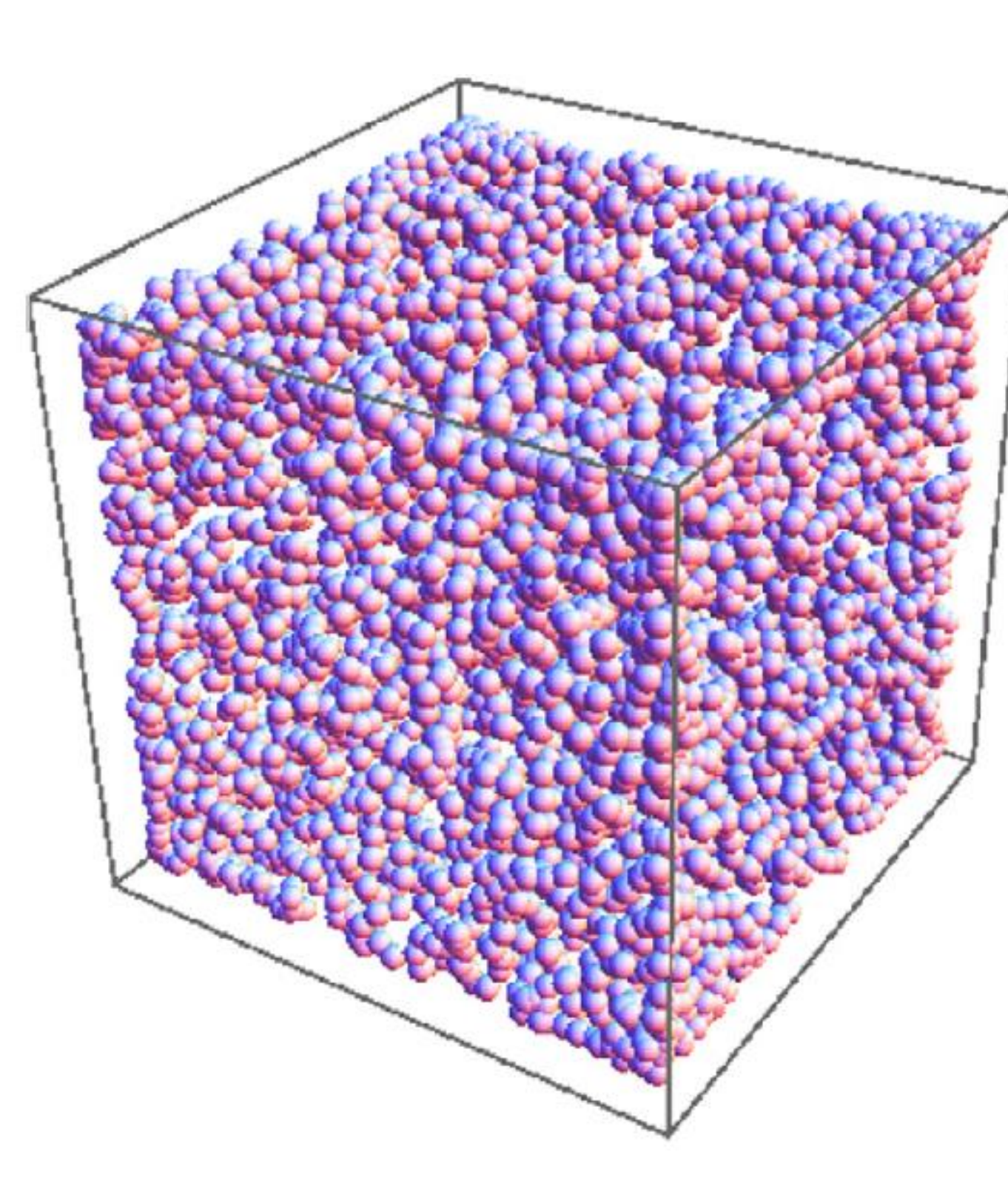

Sphere packing configuration for 5,000 spheres

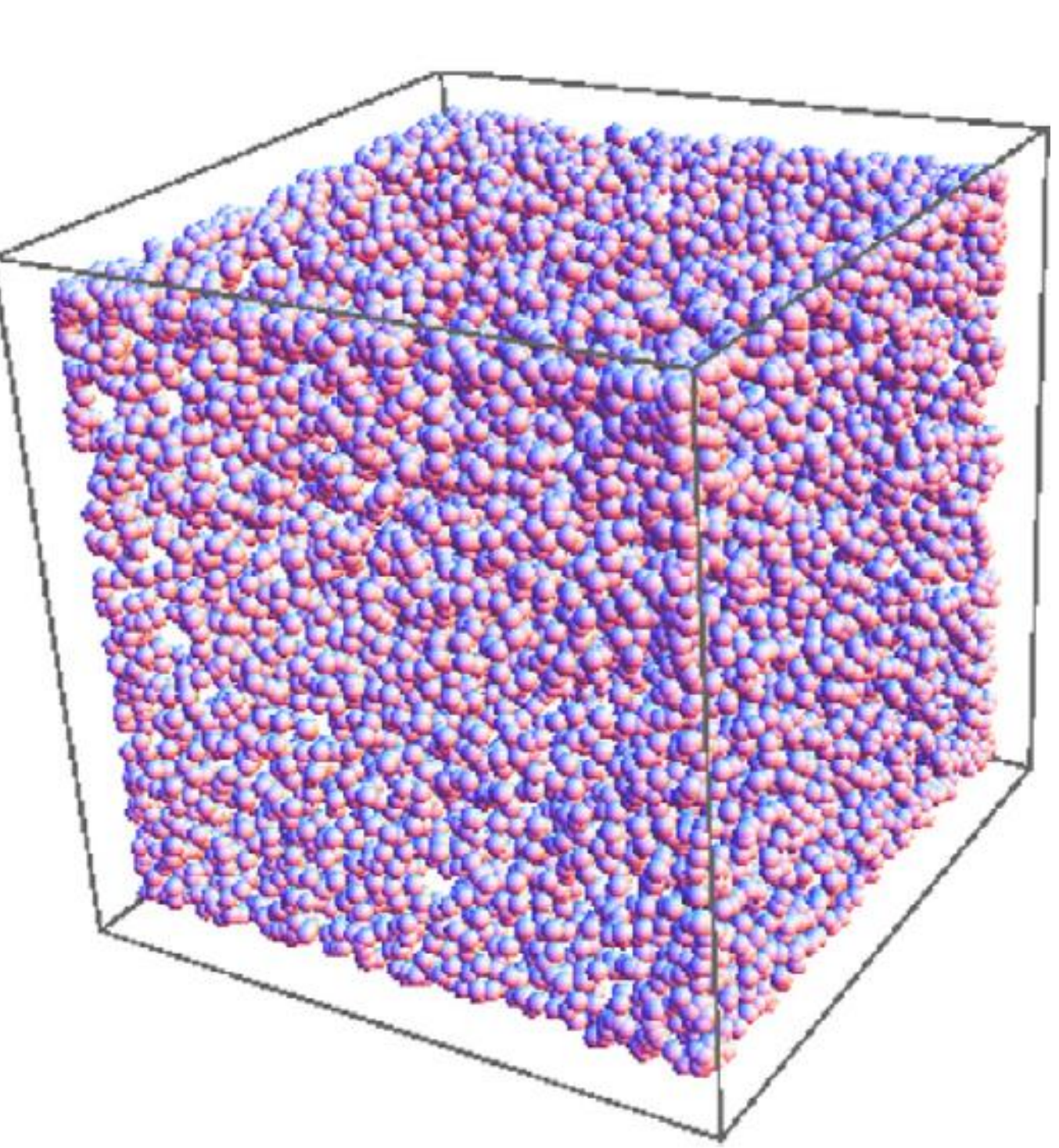

Sphere packing configuration for 10,000 spheres

\section{Conclusion}

An optimization technique called adaptive random search technique (ARSET) was employed in the sphere packing algorithm. This ARSET algorithm can vary the magnitude of random vectors based on the total potential energy difference between current and new configuration. This ARSET algorithm was proved that it can give the global minimum solution for the total potential energy. When apply this algorithm to 1000 and 1800 spheres, it gives the good agreement in solid fraction values with the results from previous works. This developed ARSET algorithm significantly reduces the actual processing time of the packing process when comparing with the basic random search technique (BRST) algorithm. The actual processing time that can be reduced using this algorithm are $5.58 \%$ for the system of 1000 spheres, $15 \%$ for the system of 1800 spheres, $22 \%$ for the system of 5000 spheres, $34 \%$ for the system of 10,000 spheres and $28.42 \%$ for the system of 30,000 spheres. The solid fractions from BRST and ARSET algorithm are very similar with the maximum percentage difference at $2.73 \%$. The effect of initial sampling range (a) was also investigated and the appropriate a value is equal to 0.5 because it showed a good performance with any number of spheres. 